\title{
COMPORTAMENTO EXPLORATÓRIO EM GATOS DOMÉSTICOS (Felis silvestris catus Linnaeus, 1758): UMA REVISÃO
}

\author{
Juliana Clemente Machado ${ }^{1}$, Gelson Genaro ${ }^{1}$ \\ 1 Universidade Federal de Juiz de Fora - juliajoe@terra.com.br
}

\begin{abstract}
RESUMO: Entende-se comportamento exploratório como uma atividade promovida por um estímulo novo e que consiste em atos e posturas que permitem a coleta de informações sobre um objeto, ou ambiente não familiar. A exploração possui fortes implicações na sobrevivência do indivíduo e na de sua espécie, ao facilitar a familiarização com situações de novidade. Este comportamento tem sido avaliado de forma mais significativa em humanos e só a partir da década de 1950 os estudos com animais não humanos começaram a ocorrer. Para gatos domésticos, a pesquisa nesta área ainda é muito tímida e a compreensão deste comportamento poderá auxiliar nas abordagens relacionadas a enriquecimento ambiental e bem-estar animal. Tendo em vista que o gato é hoje uma espécie de interesse, particularmente como animal de companhia, modelo de estudo para felinos em cativeiro e animal de experimentação, a avaliação da atual situação deste comportamento neste animal poderá auxiliar na busca de uma relação mais adequada com a sua manutenção propiciando um manejo orientado dentro de uma postura ética.
\end{abstract}

Palavras-chave: enriquecimento ambiental; exploração; felinos; neofilia; novidade

\section{EXPLORATORY BEHAVIOUR IN DOMESTIC CATS (Felis silvestris catus Linnaeus, 1758): A REVIEW}

\begin{abstract}
It is possible to understand exploratory behaviour as an activity sponsored by a new stimulus, which consists in acts and postures that enable to collect information about an object or an unfamiliar environment. The exploration has strong implications for the survival of the individual and of its specie facilitating familiarization with new situations. This behaviour has been assessed more significantly in humans and only in the 1950 studies with non-human animals began to occur. In cats, research in this area is still very shy, setting a worrying scenario as the understanding of this behaviour may help in the approaches related to environmental enrichment and animal welfare. Considering that the cat is now an animal of interest such as pet, study model for felids in general and animal experimentation, evaluation of the current situation of this behaviour can help the search for a more ethical relationship with these felids.
\end{abstract}

Key words: environmental enrichment; exploration; felids; neophilia; novelty 


\section{INTRODUÇÃO}

A exploração de ambientes e objetos não familiares tem sido descrita para uma ampla variedade de animais. Estudos com diferentes enfoques avaliam a exibição deste comportamento, em lagartos (Eulamprus heatwolei) (Stapley e Keogh, 2004), baratas (Blattela germanica) (Durier e Rivault, 2003), lagostas (Cherax destructor) (Pattulo e Macmillan, 2006), abelhas (Apis melifera) (Haupt e Klemt, 2005), roedores (Caston et al., 1998; Genaro e Schmidek, 2000; Ennaceur et al., 2009), primatas não humanos (Bergman e Kitchen, 2009), bovinos (Westerath et al.,2009), e humanos (Berlyne, 1966; Gibson,1988), propondo padrões motores de exibição, explicações psicobiológicas, interferências fármacológicas, genéticas e sociais. O estudo com animais não humanos teve destaque a partir dos anos 50, com ênfase em roedores e primatas, o que justifica o grande número de publicações com os referidos grupos em detrimento dos demais.

O comportamento exploratório pode ser definido como um conjunto de padrões motores direcionados a um ambiente ou objeto desconhecido ou pouco familiar (Berlyne, 1966; Crusio, 2001), por meio do qual informações sobre o ambiente, ou objeto, são coletadas (Hughes, 1997). Por meio deste comportamento, os animais se familiarizam com a situação de novidade aprendendo, por exemplo, onde encontrar água, alimento ou esconderijo, influenciando ainda nas habilidades competitivas (Verbeek et al., 1994). A exibição do comportamento exploratório possui importantes consequências na sobrevivência de um indivíduo e logo, na de sua espécie. As informações obtidas permitem que os indivíduos tirem vantagem da experiência exploratória, otimizando as reações futuras em qualquer situação igual, ou semelhante, maximizando as respostas em determinado ambiente (Blanchard e Cañamero, 2006). Além disso, é através deste comportamento que relações sociais podem ser estabelecidas e mantidas, evitando, por exemplo, conflitos no uso do espaço (Hughes, 1997). Os animais se expõem, portanto, a certo grau de incerteza obtendo benefícios imediatos - ao controlar 0 ambiente e reduzir a falta de familiaridade com a situação, mas também benefícios de longo prazo, questões fundamentais para a manutenção do bem estar em nível elevado (Westerath et al., 2009). Winn (2001) e Broom e Fraser (2007) afirmam que este comportamento reduz a incerteza em relação ao ambiente externo por meio da aquisição de informações sobre este, sendo instintivo e adaptativo, já que provê o organismo de informações sobre disponibilidade de alimentos, parceiros sexuais, competidores, predadores, ofertas espaciais e outros recursos.

\section{DESENVOLVIMENTO}

Autores de diferentes épocas, e campos de estudo, concordam que a exibição do comportamento exploratório só é possível devido à presença de um estímulo novo que o provoque (Berlyne, 1966; Hughes, 1997; Crusio, 2001). Berlyne (1966) define novidade como sendo um estímulo que não encontra antecedentes no passado de experiência do indivíduo e que produz orientação, atenção e exploração. Segundo este autor, a novidade pode ser absoluta, quando é totalmente desconhecida do indivíduo, ou relativa, quando alguns elementos do estímulo novo são de conhecimento do indivíduo, mas são apresentados de uma forma ainda não familiar. Sugere-se ainda que possa ser classificada em novidade 
relativa a um objeto, relativa a um ambiente, ou novidade de coespecífico, quando o estímulo novo é um indivíduo da mesma espécie nunca antes conhecido (Broom e Fraser, 2007). Tal diferenciação é importante no sentido de que existem reações diferentes e características das espécies com relação aos tipos de novidade e, dentro das espécies, estas reações podem variar com a ontogenia (Broom e Fraser, 2007). Assim esta diferenciação pode facilitar a discussão das variadas reações comportamentais dos indivíduos frente a múltiplos padrões de novidade, permitindo inclusive que se compreenda quando esta novidade é bem recebida pelo animal, ou quando, representa um grau de imprevisibilidade que gera estresse e redução do seu nível de bem-estar (Broom e Johnson, 1993).

$\mathrm{Na}$ exploração, o animal dirige ao objeto, ou ambiente, praticamente todos os padrões comportamentais a sua disposição, e é dentro deste contexto que a aprendizagem e habituação podem ocorrer (Lorentz, 1983). Inicialmente 0 animal tende a tratar a novidade com cautela, avaliando-a. Hoje sabe-se que diferentes grupos animais são capazes de avaliar a novidade quanto à forma (Yaski et al., 2008; Bergman e Kitchen, 2009), tamanho e posição (Ennaceur et al., 2009), e que fatores genéticos (Caston et al., 1998; Crusio, 2001), fisiológicos (Genaro et al., 2004), e sociais, (Dingemanse e Goede, 2001; Genaro et al., 2004; Genaro, 2005) interferem nessa avaliação. De acordo com as respostas do item ou do ambiente, outros padrões motores podem ocorrer como fuga, perseguição e perda de interesse (Lorentz, 1983).

Em diferentes estudos em laboratório, com organismos de variados grupos, tem sido notado que os animais aprendem a manejar o seu entorno, a fim de obter, como recompensa, a simples opção de explorar (Hughes, 1997). Isso demonstra que explorar é por si só, um comportamento recompensador e que a ausência de oportunidade exploratória pode ser significativamente estressante, levando a comportamentos anormais, e redução do bem-estar.

Ao afirmar que um animal precisa explorar como forma de otimizar o seu bem-estar, e reduzir o estresse, deve-se ter em mente as definições clássicas dos três conceitos: Estresse, bem-estar, e necessidade. Segundo Broom e Johnson (1993) estresse pode ser definido como um efeito ambiental sobre o indivíduo, que ultrapassa o seu sistema de controle, e reduz seu fitness. Ainda, segundo estes autores, bemestar pode ser definido como uma característica mensurável nos indivíduos, que diz respeito a sua capacidade de lidar com o próprio ambiente, e que pode variar numa escala de muito ruim a muito bom. Quanto ao conceito de necessidade, também segundo estes autores, pode-se dizer que seja algo relacionado à deficiência de um item, que seja fundamental na biologia do animal, como um recurso particular, ou estímulo físico ou ambiental. Assim, ao verificar que a exploração é a atividade que tem 0 potencial de permitir ao indivíduo adquirir novas informações de uma situação de novidade (Crusio, 2001), é possível concluir que se trata de uma necessidade (pois se relaciona a deficiência de informação sobre esta situação nova), que aumenta o bemestar (pois aumenta a capacidade dos indivíduos lidarem, de maneira ótima, com o seu entorno, reduzindo a imprevisibilidade), e que reduz o estresse (pois reduz a possibilidade de ocorrência de um efeito ambiental que ultrapasse o sistema de controle do indivíduo).

Neste contexto, a relação entre comportamento exploratório, enriquecimento ambiental, e bem estar animal 
tem sido avaliada em alguns trabalhos. Pesquisas com suínos (Sus scrofa domesticus) (Bracke e Spoolder, 2007), leopardos (Felis bengalensis) (Carlstead et al., 1993), ratos (Rattus novergicus) (Zimmermann et al., 2001), e búfalos (Westerath et al., 2009), demonstram que animais mantidos em ambientes empobrecidos exibem baixa proporção de comportamento exploratório e, na presença da novidade, mostram interação crescente e prolongada. Este fato é resultado da ausência de estímulo na vida diária destes animais, ressaltando que o ambiente empobrecido é um fator estressante que leva o animal a explorar intensamente, quando possível, como forma de recompensa. Os trabalhos demonstram também que os animais de ambientes restritos, em situação de novidade, são super responsivos ao novo e que como tiveram poucas experiências relevantes para comparar com a vivenciada, necessitam de um longo tempo para aprender sobre o objeto novo ou ambiente. Respostas mais intensas são verificadas em animais que vivem em ambientes mais pobres, já que não têm para onde direcionar a intensa motivação para explorar. Esses animais, no entanto exibem menor riqueza de atos comportamentais. A habituação ao longo do tempo é uma constante, mas, varia de acordo com a intensidade da motivação para explorar (Van de Weerd e Day, 2009) ou seja, aqueles com maior motivação exploratória, habituamse menos rapidamente.

Trickett et al., (2009) ao estudarem a habituação de porcos a dois diferentes tipos de enriquecimento ambiental, discutem que não só o grau de novidade, mas a características do estímulo determinam a atratividade, e que a aleatoriedade na apresentação reduz a habituação. Fica evidenciada então que, as características do estímulo são fundamentais para prever quão significativa, e duradoura, a resposta será. Durr e Smith (1997) ressaltam que o estímulo novo não deve ser nem tão intenso, gerando neofobia reduzindo a exploração, nem tão fraco, gerando desinteresse. As características da espécie estudada devem ser consideradas, bem como sua habilidade em percepção de forma, cores, sons, odores, e movimento, garantindo que o estimulo apresentado seja bem aceito pela espécie, não gerando estresse e, promovendo a neofilia, ser capaz de elevar o bem-estar ou mantê-lo em nível ótimo.

Dos diferentes testes utilizados para avaliar o comportamento exploratório, os mais usados são os testes de exploração forçada, em que o animal é colocado em um ambiente novo: testes de labirinto em cruz elevado, em que o animal é colocado em um aparato com quatro braços, sendo geralmente, dois abertos, e dois fechados nas extremidades; testes de exploração livre, em que o animal tem a opção de explorar um ambiente novo, ou continuar no seu familiar; e testes de exploração de um objeto em que este normalmente é introduzido no ambiente do animal (Hughes, 1997). Os testes de exploração forçada e labirinto em cruz elevado são altamente criticados na atualidade porque na maioria das vezes é impossível saber se o animal explora por motivação intrínseca de curiosidade, ou extrínseca, numa tentativa de fuga de uma condição aversiva (Chemero e Heyser, 2005). Representam ainda, uma situação ecológica muito improvável já que o animal na natureza raramente irá se deparar com uma situação de absoluta novidade, em que ele não tenha a opção de explorar, ou não. A exploração livre, e o oferecimento de um objeto não familiar, são testes mais ecologicamente válidos (Durr e Smith, 1997). Segundo Hughes (1997), a exploração de objeto não familiar é a melhor forma de avaliar o comportamento exploratório sem pos- 
síveis confusões de interpretação. A riqueza de informações pode ser obtida a partir da sequência detalhada das respostas exploratórias dos animais permitindo ainda o desenvolvimento de abordagens de aprendizado e memória. Nesta perspectiva, é possível avaliar a exibição do comportamento exploratório na introdução de objetos que combinam barulho e movimento (Durr e Smith, 1997), ou complexidade de cor e forma (Bergman e Kitchen, 2009). As características do ambiente onde 0 objeto está sendo inserido também devem ser consideradas. Harris e Knowlton (2001) demonstram, por exemplo, que coiotes (Canis latrans) cativos costumam investigar objetos novos muito mais em ambiente não familiar que no familiar, o mesmo ocorrendo para odores. A reação apresentada pelos animais é justificada pelo fato do ambiente, não familiar, ser mais rico em novidades promovendo mais exploração. A presença do objeto em um local familiar induz a ansiedade de não se conhecer parte da discrepância espacial que 0 objeto significa, e a situação é tratada com cautela.

Chemero e Heyser (2005) defendem enfaticamente o uso de objetos no estudo do comportamento exploratório. Segundo os autores duas vantagens podem ser percebidas no estudo com exploração de objetos. Primeiro não é necessário privar o animal de nenhum recurso básico como alimento e água, o que oferece uma observação da exploração por si mesma. E segundo, possibilita o estudo de outros temas como habituação, memória e aprendizado. Os autores mostram que pouca ou nenhuma atenção tem sido dada a descrever as características dos objetos avaliados, e por vezes, objetos de característica muito diferentes (em forma, tamanho e textura, por exemplo), são comparados sem qualquer critério. Os autores aconselham que o objeto deva suportar o peso do animal, deva ficar paralelo ao solo e deva ter distância, largura e altura proporcionais ao corpo do animal estudado.

Muitos trabalhos abordando enriquecimento ambiental, embora não objetivem diretamente a avaliação do comportamento exploratório, têm utilizado a exibição deste como parâmetro para avaliar a eficiência do enriquecimento em questão, ou a supressão deste comportamento como indicativo de estresse. Tem-se utilizado novidades áudio-visuais como televisões para gatos (Ellis e Wells, 2008), espelhos para golfinhos (Tursiops truncatus), e elefantes (Elephas maximus) (Reiss e Marino, 2001; Plotnik et al., 2006), e computadores para primatas não humanos (Platt e Navak, 1997), a fim de estimular a exploração de diferentes grupos animais. Sons de diferentes fontes, como sons da natureza, para jaguatiricas, e música clássica para cães, também têm sido empregados (Powell, 1997; Wells, 2004). Todas essas novas formas de estimulação sensorial têm obtido significativos resultados na redução de comportamentos estereotipados, automutilação e inatividade. De maneira geral a exibição do comportamento exploratório tem sido uma ferramenta na demonstração da efetiva utilidade de um determinado item de enriquecimento, já que a maioria dos testes é feita permitindo ao animal optar por explorar, ou não. Além dos já referidos estímulos visuais e sonoros, formas diferenciadas de apresentação de alimentos, estímulos cognitivos, e estímulos odoríferos têm sido utilizados como forma de aumentar a complexidade de um determinando ambiente de cativeiro (Wells et al., 2006).

O comportamento exploratório dos gatos domésticos não tem sido avaliado de forma significativa no meio científico e os poucos trabalhos que relatam este 
comportamento possuem o enfoque voltado para relações dominância (Durr e Smith, 1997; Crowell-Davis et al., 2004), relações mãe e filhote (Bateson, 2000; Deag et al., 2000), e comportamento alimentar (Fitzgerald e Turner, 2000). Trabalhos com enriquecimento ambiental também tem utilizado o comportamento exploratório como medida de atividade (Ellis, 2007). Tanto o estudo com gatos domésticos quanto as pesquisas com comportamento exploratório têm um histórico recente, o que justifica a dificuldade em se encontrar na literatura trabalhos com este comportamento voltado para estes felinos, como tem sido feito para outros grupos animais, principalmente roedores e primatas. Considerando o papel do gato doméstico na sociedade atual, e também no meio científico e, tendo em vista 0 crescimento das temáticas ligadas ao comportamento exploratório, é de esperar que ao longo dos anos a avaliação aprofundada deste comportamento, para este grupo se efetue, com vistas à melhor compreensão do repertório comportamental destes felinos, e aplicação do conhecimento no oferecimento de condições que permitam a melhoria do seu bem-estar,

Em 1977, West avaliou o comportamento exploratório de filhotes de gatos domésticos, e o descreveu da seguinte maneira: "A exploração gira em torno da percepção visual, atração, olfação e/ou touque, sem a manipulação efetiva do objeto, com patas ou boca, enquanto lentamente move-se em direção, ou em volta do objeto." Tilson e Seal (1987) defendem que os felinos de uma maneira geral, tendem a ser muito mais neofílicos que neofóbicos, o que pode ser justificado pelo papel de predadores que apresentam. Hosey (2008), ao avaliar as relações entre o homem e os animais em zoológico, verifica que para felinos, muitas vezes 0 efeito da qualidade do recinto é muito mais perceptível em seu comportamento do que a interferência da presença dos visitantes. Já para primatas, o efeito parece ser contrário, com os visitantes exercendo intenso papel no comportamento exibido. De acordo com a pobreza do recinto, com impossibilidade de exploração e fuga, esconderijos, e sem a presença de itens que estimulem a atividade, felinos tendem a apresentar alto grau de estereotipias $e$ inatividade (Tilson e Seal, 1987). Isto demonstra quão significativo para este grupo animal é o oferecimento de oportunidades de exibição do seu repertório comportamental, melhorando a qualidade do ambiente. Esta melhoria deve versar desde fatores simples, como a textura do assoalho, e local para abrigo, como questões mais complexas como: itens que estimulem a capacidade cognitiva dos animais, e que promovam a exploração da área.

Dos poucos trabalhos que lidam com o comportamento exploratório de forma mais central na família Felidae, merecem destaque os trabalhos de Glickman e Sroges (1968), Carlstead et al., (1993) e Durr e Smith (1997), bem como os trabalhos de Wells e Egli (2004) e Ellis (2007).

Glickman e Sroges (1968) desenvolveram um interessante trabalho em que analisaram a curiosidade em animais de zoológico, com a introdução de objetos não familiares que variassem textura, forma e odor. Os resultados demonstram diferenças significativas entre vários grupos taxonômicos, tanto na quantidade quanto na forma de manipulação dos itens. Os autores concluíram que os Primatas, e os Carnívoros, foram os que exibiram maior comportamento de investigação; os animais jovens tendem a ser mais reativos que os adultos, e que os pequenos felinos do gênero Felis foram menos reativos que os grandes felinos do gênero Panthera. Com relação a sequências motoras, a principal reação 
da maioria dos animais foi morder o item, como esperado. Além disso, os primatas seguram 0 item com os membros anteriores, inspecionando visualmente, e manipulando o item. Os Carnívoros mostraram uma aproximação vigorosa, com poucos indícios de medo, com um padrão motor que se assemelha ao normalmente utilizado na obtenção de presas: caminhar lenta, e silenciosamente, em direção ao objeto, exibir comportamentos típicos de perseguição, morder, atacar, e se colocar sobre as patas. Algumas possíveis técnicas de exploração típicas das espécies foram notadas, como o uso da língua pelas antas (Tapirus terrestris) e da cauda pelo macaco aranha (Ateles geoffroyi). Os autores remetem essas diferenças comporta-mentais a padrões alimentares, posição ecológica de presa ou predador e correlações do desenvolvimento neurológico.

Carlstead et al., (1993) conduziram um trabalho com enriquecimento ambiental no recinto de gatos leopardos (Felis bengalensis), no zoológico nacional de Washington/EUA, e as relações desse enriquecimento com suas respostas adrenocorticais e comportamentais. Por meio de enriquecimento composto por galhos, e locais de esconderijo, os níveis de cortisol urinário, e o comportamento estereotipado de "pacing", reduziram significativamente, enquanto que 0 comportamento exploratório aumentou de forma considerável. Os autores sugerem que reduzida exibição de comportamento exploratório denuncia a exposição crônica a condições ambientais aversivas, e que os programas eficientes de enriquecimento ambiental devem estimular 0 comportamento em questão. Assim, fica evidenciada a abordagem "bemestar animal" no estudo deste comportamento.
Durr e Smith (1997) realizaram um dos raros trabalhos que enfocam a resposta de gatos domésticos a estímulos de novidade. Os autores utilizaram dois grupos de animais com ordens sociais bem estabelecidas, compostos de machos e fêmeas não castrados. Segundo os autores, o melhor estímulo de novidade a ser oferecido não deve ser tão intenso para não afastar os animais, nem tão suave para não se correr o risco de não chamar a atenção dos mesmos. Assim os autores associaram movimento, e barulho, em um objeto que foi introduzido no recinto desses animais em condição natural. Outros estímulos e condições também foram testados como alimento e exploração individual. O objetivo era relacionar grau de dominância social e diferenças individuais na exploração dos itens. Avaliando a latência em dirigir a atenção ao objeto e o contato com o mesmo, os autores concluíram que os animais demonstraram diferenças individuais na reação a novidade que foi independente do grau de dominância que esses exibiam no grupo, ou seja, o animal dominante nem sempre o é em todas as situações, ou tipos de atividade. Ao contrário de lobos (Canis lupus) (Svartberg, 2005) os animais dominantes, no ranking social e na competição alimentar, não foram os mais neofílicos, e tal diferença é, segundo os autores, derivada de diferenças nas características sociais das duas espécies, bem como no grau de domesticação.

Sabendo-se que o olfato é talvez o sentido mais desenvolvido em gatos domésticos, alguns autores têm direcionado suas pesquisas para comportamento exploratório, enriquecimento ambiental e bem-estar, na questão dos odores. Assim que ocorre o parto é o olfato que irá orientar o animal em direção às mamas da mãe (Beaver, 1992). Além disso, o odor é utilizado na 
identificação entre gatos, bem como na exploração, marcação e habituação com novos ambientes (Turner e Bateson, 2000). Qualidades sociais também podem ser percebidas. (Beaver, 1992; Turner e Bateson, 2000). Assim, podese verificar que a introdução de odores pode ser um interessante meio de promover a atividade de avaliação e identificação de um território, ou objeto, promovendo o comportamento exploratório em felinos domésticos.

Wells e Egli (2004) realizaram um trabalho avaliando a influência de um enriquecimento olfatório (representado por noz moscada, catnip, e odor de presa - codorna) no comportamento do gato de patas negras (Felis nigripes) em cativeiro. Os odores foram adicionados a uma flanela, e esta foi apresentada por 5 dias no recinto dos animais. Os odores estimularam os animais a passar menos tempo exibindo comportamentos de inatividade, aumentando o comportamento exploratório, e a locomoção. A noz moscada exerceu a menor influência no comportamento que odores de presa e catnip. Houve habituação com os estímulos ao longo dos dias, e os autores sugerem alternar a apresentação, ou oferecê-los ocasionalmente. De maneira geral, os odores aumentaram a atividade dos gatos cativos, reduzindo o sedentarismo, e encorajando a exploração, mudanças que são desejáveis dentro da perspectiva do bem-estar animal.

Ellis (2007) avaliou em 150 gatos, de ambos os sexos, o efeitos de enriquecimento odorífero com lavanda, catnip e odor de presa (coelho). Verificou que os animais demonstraram exploração ativa principalmente na presença do odor de catnip e de presa, embora os demais comportamentos exibidos fossem relacionados com a inatividade. A autora justifica a exibição significativa de inatividade, com base no fato de que eram animais provenientes de cidades, que provavelmente não possuíam interesse no odor de uma presa que não conheciam. O trabalho foi realizado no período da tarde, o que também pode justificar $o$ índice de inatividade observado.

Para espécies predadoras como gatos, a exploração é uma importante parte na localização de alimento, e logo, a novidade (na forma de odores) pode aumentar a motivação para explorar (Ellis, 2007). Broom e Fraser (2007) defendem ainda que todos os animais domésticos têm uma forte motivação para explorar quando encontram a novidade. Gatos reconhecem suas presas principalmente através da visão e da audição (Jensen, 2002), mas não podemos deixar de considerar, no entanto, que sendo um animal responsivo a estimulação odorífera, o odor da presa pode ser uma forma significativa de estimulação, com facilidade de aplicação em ambientes cativos, por aqueles que objetivem aumentar a taxa exploratória do animal, em direção a melhoria da sua qualidade de vida, e elevação do seu nível de bem-estar.

\section{CONCLUSÃO}

A tendência da pesquisa etológica com animais tem sido a de conhecer comportamentos básicos e aplicar este conhecimento, principalmente, na melhoria da qualidade de vida dos indivíduos sejam estes domésticos, selvagens, ou de criação, cativos, ou de vida livre. Verificamos ainda uma lacuna significativa na compreensão do comportamento exploratório dos animais, como um todo, e enfaticamente, para gatos domésticos. Esta defasagem reforça a manutenção dos animais em situação não ideal prejudicando o seu bem-estar, e indo contra a abordagem ética que devemos ter com os demais seres vivos. Novas técnicas que objetivem a melhoria da manutenção dos gatos cativos devem 
ser propostas, tendo em vista todas as questões discutidas nesta revisão como a importância da novidade, a influência dos odores e a possibilidade da neofobia, a fim de que, no futuro, possamos manter gatos cativos sadios nas diversas condições (experimentais, domésticas, cativeiro) não só na perspectiva sanitária, mas também comportamental.

\section{AGRADECIMENTOS}

Os autores agradecem a agência de fomento a pesquisa CAPES, a Empresa Laboratórios DUPRAT ${ }^{\circledR}$, e a médica Maria José Ribeiro Toledo por apoiarem este trabalho.

\section{REFERÊNCIAS}

BATESON, P. Behavioral development in the cat. In: TURNER, D.C.; BATESON, P. The domestic cat: The Biology of its Behaviour. Cambridge: Cambridge University Press, 2000, cap.2, p.9-21.

BEAVER, B.V. Feline Behavior: A guide for veterinarians. Saunders, 1992. 276p.

BERGMAN, T.J.; KITCHEN, D.M. Comparing responses to novel objects in wild baboons (Papio ursinus) and geladas (Theropithecus gelada). Animal Cognition, v.12, n.1, p.63-73, 2009.

BERLYNE, D.E. Curiosity and exploration. Science, n.153, p.25-33, 1966.

BLANCHARD, A.J.; CAÑAMERO, L. Modulation of exploratory behavior for adaptation to the context. In: SYMPOSIUM ON BIOLOGICALLY INSPIRED ROBOTICS,6, 2006, Bristol.

Proceedings ... Bristol: UK, 2006. p.131-137.

BRACKE, M.B.M.; SPOOLDER, A.M. Novel object test can detect marginal differences in environmental enrichment in pigs. Applied Animal Behavior Science, v.109, n.1, p. 39-48, 2007.

BROOM, D.M.; JOHNSON, K.G. Stress and animal welfare. London: Chapman e Hall, 1993. $211 p$.

BROOM, D.M.; FRASER, A.F. Domestic Animal Behaviour and Welfare. Wallingford, UK: CABI Publishing, 2007. 438p.
CARLSTEAD, K.; BROWN, J.L;

SEIDENSTICKER, J. Behavioral and adrenocortical responses to environmental changes in leopard cats (Felis bengalensis). Zoo Biology, v.12, n.3, p. 321-331, 1993.

CASTON, J.; CHIANALE, C., DELHAYEBOUCHAUD, N. et al. Role of the cerebellum in exploration behavior. Brain Research, v.808, n.2, p.232-237, 1998.

CHEMERO, A.; HEYSER, C. Object exploration and a problem with reductionism. Synthese, v.147, n.3, p.403-423, 2005.

CROWELL-DAVIS, S.L.; CURTIS, T.M.; KNOWLES, R.J. Social organization in the cat: A modern understanding. Journal of Feline Medicine Surgery, v.6, n.1, p.19-28, 2004.

CRUSIO, W.E. Genetic dissection of mouse exploratory behaviour. Behavioral Brain Research, v.125, n.1-2, p.127-132, 2001.

DEAG, J.M.; MANNING, A.; LAWRENCE, C.E. Factors influencing the mother-kitten relationship. In: TURNER, D.C.; BATESON, P. In: The domestic cat: The Biology of its Behaviour. Cambridge: Cambridge University Press, 2000, cap.3, p.23-29.

DINGEMANSE, N.J.; GOEDE, P. The relation between dominance and exploratory behavior in context- dependent in wild great tits. Behavioral Ecology, v.15, n.6, p.1023-1030, 2001.

DURIER, V.; RIVAULT, C. Exploitation of home range and spatial distribution of resources in German cockroach (Dictyoptera: Blattellidae). Journal of Economic Entomology, v.96, n.6, p. 1832-1837, 2003.

DURR, R.; SMITH, C. Individual differences and their relation to social structure in domestic cats. Journal of Comparative Psychology, v.111, n.4, p. 412-418, 1997.

ELLIS, S.L.H. Sensory enrichment for cats (Felis silvestris catus) housed in an animal rescue shelter. 2007. UK. Ph.D. Thesis. Queen's University Belfast - UK.

ELLIS, S.L.H.; WELLS, D.L. The influence of visual stimulation on the behaviour of cats housed in a rescue shelter. Applied Animal Behaviour Science, v.113, n.1, p. 166-174, 2008.

ENNACEUR, A.; MICHALIKOVA, S.; CHAZOT, P.T. Do rats really express neophobia towards novel objects? Experimental evidence from exposure to novelty and to an object recognition task in an open space and an enclosed space.

Behavioural Brain Research, v.197, n.2, p.417434, 2009. 
FITZGERALD, M.B.; TURNER, D. Hunting behaviour of domestic cats and their impact on prey populations. In: TURNER, D.C E BATESON, P. The domestic cat: The Biology of its Behaviour. Cambridge: Cambridge University Press, 2000, cap.8, p.152-175.

GENARO, G.; SCHMIDEK, W.R Exploratory activity of rats in three different environments. Ethology, v.106, n.9, p.849-859, 2000.

GENARO G.; SCHMIDEK, W.R.; FRANCI, C.R. Social condition affects hormone secretion and exploratory behavior in rats. Brazilian Journal of Medical and Biological Research, v.37, n.6, p.833-840, 2004.

GENARO, G. Exploratory behavior of female rats born to differently raised mothers. Revista Brasileira de Zoociências, v.4, n.1, p.111-120, 2005.

GIBSON, E.J. Exploratory behavior in the development of perceiving, acting, and the acquiring of knowledge. Annual Review of Psychology, v.39, p.1-41, 1988.

GLICKMAN, S.E.; SROGES, R.W. Curiosity in zoo animals. Behavior, v.26, n.1-2, p.151-188, 1968.

HARRIS, C.E.; KNOWLTON, F.F. Differential responses of coyotes to novel stimuli in familiar and unfamiliar settings. Canadian Journal of Zoology, v.79, n.11, p.2005-2013, 2001.

HAUPT, S.S.; KLEMT, W. Habituation and dishabituation of exploratory and appetitive responses in the honey bee (Apis mellifera L.).

Behavioural Brain Research, v.165, n.1, p.1217, 2005.

HOSEY, G. A preliminary model of humananimal relationships in the zoo. Applied Animal Behaviour Science, v.109, n.2-4, p.105-127, 2008.

HUGHES, R.N. Intrinsic exploration in animals: motives and measurement. Behavioural Process, v.41, n.3, p.213-226, 1997.

JENSEN, P. The Ethology of Domestic Animals: an Introductory Text. Wallingford, U.K: CABI Publishing, 2002. 246p.

LORENTZ, K.Z. The foundations of ethology. New York: Simon e Schuster, 1983. 404p.

PATTULO, B.W.; MACMILLAN, D.L. Corners and bubble wrap: the structure and texture of surfaces influence crayfish exploratory behaviour. The Journal of experimental Biology, v.209, n.3, p.567-575, 2006.

PLATT, D.M.; NOVAK, M.A. Video stimulation as enrichment for captive rhesus monkeys (Macaca mulatta). Applied Animal Behaviour Science, v.52, n.1, p.139-155, 1997.

PLOTNIK, J.M.; WAAL, F.B.M.; REISS, D. Selfrecognition in an Asian elephant. Proceedings of the National Academy of Sciences, v.103, n.45, p.1753-1757, 2006.

POWELL, K.E. Environmental enrichment program for Ocelots at North Carolina Zoological Park, Asheboro. International Zoo Yearbook, v.35, n.1, p.217-224, 1997.

REISS, D.; MARINO, L. Mirror self-recognition in the bottlenose dolphin: a case of cognitive convergence. Proceedings of the National Academy of Sciences, v.98, n.10, p.59375942, 2001.

STAPLEY, J.; KEOGH, J.S. Exploratory and antipredator behaviours differ between territorial and non-territorial male lizards. Animal

Behavior, v.68, n.4, p.841-846, 2004.

SVARTBERG, K. A comparison of behaviour in test and in everyday life: evidence of three consistent boldness-related personality traits in dogs. Applied Animal Behaviour Science, v.91, n.1, p.103-128, 2005.

TILSON, R.L.; SEAL, S.U. Tigers of the world: the biology, biopolitics, management and conservation. New Jersey: Park Riedge, Noyes Publications, 1987. 510p.

TRICKETT, S.L.; GUY, J.H.; EDWARDS, S.A. The role of novelty in environmental enrichment for the weaned pig. Applied Animal Behaviour Science, v.116, n.1, p.45-51, 2009.

TURNER, D.C.; BATESON, P. The domestic cat: The biology of its behaviour Cambridge: Cambridge University Press, 2000.244p.

VAN DE WEERD, H.A.; DAY, J.E. A review of environmental enrichment for pigs housed in intensive housing systems. Applied Animal Behaviour Science, v.116, n.1, p.1-20, 2009.

VERBEEK, M.E.M.; DRENT, P.J.; WIEPKEMA, P.R. Consistent individual differences in early exploratory behaviour of male great tits. Animal Behaviour, v.48, n.5, p.1113-1121, 1994.

WELLS, D.L. A review of environmental enrichment for kennel dogs, Canis familiaris. Applied Animal Behaviour Science, v.85, n.3, p.307-317, 2004.

WELLS, D.L.; EGLI, J.M. The influence of olfactory enrichment on the behaviour of blackfooted cats, Felis nigripes. Applied Animal Behaviour Science, v.85, n.1, p.107-119, 2004. WELLS, D.L.; COLEMAN, D.; CHALLIS, M.G. A note on the effect of auditory stimulation on the behaviour and welfare of zoo-housed gorillas. 
Applied Animal Behaviour Science, v.100, n.3, p.327-332, 2006.

WEST, M.J. Exploration and play with objects in domestic kittens. Developmental

psychobiology, v.10, n.1, p.53-57, 1977.

WESTERATH, H.S.; LAISTER, S.; WINCKLER, C. et al. Exploration as an indicator of good welfare in beef bulls: An attempt to develop a test for on-farm assessment. Applied Animal Behaviour Science, v.116, n. 2, p.126-133, 2009.

WINN, P. Dictionary of biological psychology. Routledge, 2001. 857p.
YASKI, O.; EILAM, D.O. How do global and local geometries shape exploratory behavior in rats? Behavioral Brain Research, v.187, n.2, p.334342, 2008.

ZIMMERMANN, M.; STAUFFACHER, W.; LANGHANS, W. et al. Enrichment-dependent differences in novelty exploration in rats can be explained by habituation. Behavioral Brain Research, v.121, n.1-2, p.11-20, 2001. 